\title{
Progetto per uno studio pilota per un ambulatorio di nefrologia narrativa: un approccio bio-psico-sociale alla malattia renale cronica
}

\author{
Alessandro Toccafondi ${ }^{1}$, Leonardo Mari ${ }^{2}$, Umberto M.S. Caraccia ${ }^{3}$, Silvia Lapini ${ }^{4}$, Pietro C. Dattolo ${ }^{5}$, Claudia Capanni ${ }^{6}$, \\ Stefania Polvani' ${ }^{7}$ Marco Lombardi ${ }^{8}$ \\ ${ }^{1}$ SOSD Psicologia Clinica in servizio presso SOC Nefrologia e Dialisi Firenze 2 ASLTC, Firenze - Italia \\ ${ }^{2}$ Farmacia, Ospedale Borgo San Lorenzo, USL Toscana Centro, Firenze - Italia \\ ${ }^{3}$ Coordinatore Ambulatorio di Medicina Narrativa ASL Rieti, Rieti - Italia \\ ${ }^{4}$ SOSD Psicologia Clinica, ASLTC, Firenze - Italia \\ ${ }^{5} \mathrm{SOC}$ Nefrologia e Dialisi Firenze 2, ASLTC, Firenze - Italia \\ ${ }^{6}$ Direzione Sanitaria Firenze 2, ASLTC, Firenze - Italia \\ ${ }^{7}$ Sociologa, Azienda USL Toscana Sud-Est, Presidente Società Italiana di Medicina Narrativa (SIMeN), Arezzo - Italia \\ ${ }^{8}$ SOS Nefrologia e Dialisi, ASLTC Ospedale del Mugello, Borgo San Lorenzo, Firenze - Italia
}

\begin{abstract}
Project for a Pilot Study for a Narrative Nephrology Clinic: A Bio-Psycho-Social Approach to Chronic Kidney Disease Introduction: Poor medication adherence in CKD patients is associated with high mortality and morbidity and can reach up to $80 \%$. The most effective interventions to increase patients' adherence to treatments are conducted by a multi-professional team and with the active participation of patients and their families. Narrative evidencebased medicine has been proved as a useful methodology in the care of chronic patients also in promoting their adherence to treatments.

Methods: Around $50-70 \%$ of CKD patients treated in our centre will follow clinical protocol based on a narrative medicine approach: (1) patients will be invited to write a brief history related to their experience with the CKD and treatments; (2) screening for depression (HADS; PHQ-9); (3) intervention of pharmaceutical counselling aimed to explore patients' behaviours about taking their medications; (4) sharing of decision-making: the results of the narrative histories and of questionnaires will be discussed with the patients; (5) psychoeducational groups.

Results: The pilot phase of the project will last 6 months. During these months, patients will be monitored for biochemical (e.g. anemia) and anthropometric (e.g. blood pressure) parameters and depression. Medical visits or diagnostic tests missed by patients will also be recorded.

Conclusions: The development of an active role of patients in taking care of themselves is one of the main challenge of clinical nephrology. The present project aims to implement in the clinical routine a protocol based on the narrative medicine approach in which the biological, psychological and social needs of CKD patients are considered and discussed with them.
\end{abstract}

Keywords: Depression, Narrative medicine, Patient's compliance, Pre-dialysis, Quality of Life

\section{Introduzione}

La malattia renale cronica (MRC) è definita come una condizione di alterata funzione renale che persiste per più di 3

Received: March 21, 2020

Accepted: March 30, 2020

Published online: April 24, 2020

Indirizzo per la corrispondenza:

Marco Lombardi

Nefrologia e Dialisi Ospedale Nuovo del Mugello

Via della Resistenza 60

Borgo San Lorenzo

50032 Firenze - Italia

lombardim@tin.it mesi ed è classificata in 5 stadi di crescente gravità, che vanno dalla patologia lieve (che necessita di terapia conservativa) fino all'assenza di funzionamento dell'organo, in cui spesso è necessaria la terapia sostitutiva (dialisi e trapianto, stadio $5 \mathrm{~d}$ ). Gli studi internazionali mostrano una prevalenza della MRC nella popolazione adulta che varia dal $6 \%$ al $10 \%(1)$, con un trend che appare in aumento. Le stime di prevalenza in Italia sono in linea con questi dati $(2,3)$.

È stato dimostrato che una diagnosi corretta e precoce e una terapia adeguata possano ritardare la progressione della malattia renale verso l'End-Stage Renal Disease (ESRD), arrestare l'evoluzione $\mathrm{o}$, addirittura, in alcuni casi, portare a vari gradi di regressione della patologia (4). Anche dal punto di 
vista economico, la possibilità di intervenire precocemente si tradurrebbe in un notevole risparmio, considerati gli alti costi della MRC, che variano da 10.000 euro anno/paziente per gli stadi 4 e 5 (5) (pazienti ancora in terapia conservativa) fino a 30.000-40.000 euro anno/paziente per la dialisi peritoneale e oltre 50.000 euro anno/paziente per i pazienti in emodialisi (6); i pazienti trapiantati hanno un costo di 80.000 euro il primo anno e di 10.000 euro gli anni successivi (7).

La persona affetta da MRC va indirizzata a un percorso diagnostico-terapeutico che si basa sul miglioramento degli stili di vita (in particolare dieta a basso contenuto di sale, con l'apporto proteico consigliato dalle Linee Guida nazionali per una sana alimentazione) e sul trattamento delle eventuali patologie concomitanti (8). Per il raggiungimento di questi scopi, il coinvolgimento attivo del paziente nel percorso di cura e l'aderenza ai trattamenti identificati sono due elementi essenziali (9).

La scarsa aderenza alle prescrizioni mediche è una delle più importanti problematiche nella cura nei pazienti cronici in generale e con MRC in particolare ed è un elemento chiave della gestione del percorso diagnostico-terapeutico (10). La letteratura dimostra che la mancata aderenza al piano terapeutico concordato con il nefrologo può arrivare fino all' $80 \%$ dei pazienti nefropatici $(11,12)$. La non aderenza è associata a una mortalità e a una morbilità elevate $(12,13)$ e costituisce un criterio negativo nella valutazione per l'inserimento nella lista d'attesa di trapianto. Inoltre, incrementa i costi per l'assistenza sanitaria in termini di maggiori esami diagnostici, frequenti accessi al Pronto Soccorso, dialisi in emergenza, degenze ospedaliere e attività del personale sanitario. Ottimizzare l'aderenza alle prescrizioni mediche è, quindi, una priorità assoluta in questo campo della salute (9).

Per favorire l'aderenza alle cure nella MRC, come in altre malattie croniche, è essenziale implementare interventi di alfabetizzazione sanitaria e di educazione terapeutica per il paziente e i suoi familiari. La letteratura internazionale riporta vari modelli di intervento; le esperienze più promettenti risultano essere quelle che vedono il coinvolgimento di un team strutturato e multi-professionale e la partecipazione attiva non solo del paziente ma anche dei suoi familiari $(8,14)$.

Uno dei fattori maggiormente associati alla mancata aderenza alle cure nei pazienti nefropatici è la depressione $(15,16)$, che rappresenta la psicopatologia più frequente nei pazienti affetti da MRC, interessando fino al $20-30 \%$ di questa popolazione di malati (17). Dal momento che i pazienti depressi hanno tre volte in più di probabilità di non aderire al percorso di cura rispetto ai pazienti non depressi (18), la prevenzione e il trattamento precoce dei disturbi depressivi, attraverso uno screening costante del distress psicologico e interventi psicologici specifici, possono incidere positivamente sull'aderenza del paziente al percorso di cura, migliorando, infine, i risultati clinici (19).

Sono numerosi gli aspetti che ostacolano l'aderenza alle cure; l'Organizzazione Mondiale della Sanità riporta come questa sia ostacolata da una pluralità di fattori: sociali, economici e legati all'organizzazione dei servizi sanitari, alla relazione con i curanti, alle caratteristiche della malattia e delle terapie e a fattori psicosociali del paziente. Per intervenire efficacemente sul problema dell'aderenza alle cure sono necessari, pertanto, interventi multidisciplinari sul piano medico, psicologico e sociale, personalizzati in base alle specifiche problematiche dei pazienti, in modo da migliorare la relazione di cura e, di conseguenza, l'aderenza terapeutica (20).

\section{Il progetto pilota}

II seguente progetto intende realizzare un intervento clinico per promuovere l'aderenza dei pazienti al percorso di cura. L'ambulatorio di Nefrologia Narrativa vuole essere un servizio integrato di umanizzazione e di etica delle cure, per i pazienti afferenti alla SOS Nefrologia e Dialisi dell'Ospedale del Mugello afferente alla SOC di Nefrologia e Dialisi 2, dell'Azienda USL Toscana Centro. L'attività che si intende svolgere farà riferimento alle "Linee di indirizzo per l'utilizzo della Medicina Narrativa in ambito clinico-assistenziale" secondo il Documento di Consenso dell'Istituto Superiore di Sanità, dove sono state espresse, secondo l'Evidence-Based Medicine, definizioni e metodologie Narrative-Based Medicine (NBM), e alla successiva letteratura in merito.

\section{Target}

Pazienti afferenti all'ambulatorio di pre-dialisi in trattamento nefrologico perché affetti da MRC in fase pre-dialitica.

\section{Sede del progetto e personale coinvolto}

SOS Nefrologia e Dialisi Ospedale Borgo San Lorenzo, afferente alla SOC Nefrologia e Dialisi Firenze 2 diretta dal Dottor Pietro C. Dattolo. II personale coinvolto è indicato nella Tabella I.

Tabella I - Personale coinvolto

\section{Referenti}

Dottor Pietro Claudio Dattolo - Direttore SOC Nefrologia e Dialisi Firenze 2

Dottoressa Silvia Lapini - Direttore SOSD Psicologia Clinica

Dottor Marco Lombardi - Direttore SOS Nefrologia e Dialisi Ospedale Borgo San Lorenzo

\section{Personale necessario per lo svolgimento del progetto}

Dottor Marco Lombardi - SOS Nefrologia e Dialisi Ospedale Borgo San Lorenzo

Dottor Alessandro Toccafondi - SOSD Psicologia Clinica, in servizio presso SOC Nefrologia e Dialisi Firenze 2

Dottor Leonardo Mari - SOS Farmacia Ospedale Borgo San Lorenzo

Dottor Umberto Mauro Salvatore Caraccia - Psicologo Coordinatore Ambulatorio Medicina Narrativa ASL Rieti 


\section{Obiettivi - fase di sperimentazione}

Nella fase sperimentale l'obiettivo generale è valutare la fattibilità del progetto in termini di integrazione dell'attività nella routine clinica e l'aderenza dei pazienti alle attività proposte.

Obiettivi specifici:

- maggiore aderenza alle cure

- miglioramento della qualità di vita

- riduzione della sintomatologia depressiva clinicamente rilevante

- contenimento della progressione della MRC, delle sue complicanze e della progressione delle usuali comorbilità associate

\section{Durata}

Fase di sperimentazione: 6 mesi.

\section{Metodologia}

Una quota stimata in almeno il 50-70\% degli oltre 100 pazienti afferenti all'ambulatorio di pre-dialisi dell'Ospedale Borgo San Lorenzo riceverà il seguente protocollo clinico (Fig. 1):

1) una prima visita medica alla presenza del nefrologo e di uno psicologo. Durante tale visita, laddove se ne ravvedano la possibilità e l'utilità, al paziente sarà chiesto di scrivere e riportare alle visite successive una breve storia relativa ai vissuti soggettivi legati alla sua malattia e al relativo percorso di cura. I vissuti, analizzati secondo procedure e nodi di analisi di medicina narrativa basata sull'evidenza, daranno luogo a dati che saranno utili per rendere le modalità assistenziali più idonee, più appropriate e più efficaci sul piano medico, psicologico e sociale e sul piano dell'umanizzazione delle cure. La cartella clinica del paziente sarà integrata (cartella parallela) con le informazioni raccolte dall'analisi delle narrazioni di malattia (storie scritte dai pazienti e analisi ottenute). Potranno avere accesso a tali informazioni tutti i sanitari coinvolti nella cura del paziente, compreso il suo MMG, secondo le norme vigenti sulla privacy, oltre che il paziente, qualora ne faccia richiesta;

2) screening per la depressione. Durante la prima visita medica, a tutti i pazienti sarà somministrato il Patient Health Questionnaire-9 (PHQ-9) o l'Hospital Anxiety and Depression Scale (HADS). I pazienti che otterranno un punteggio sopra il cut-off saranno invitati a una sessione individuale con lo psicologo;

3) intervento di counselling farmaceutico. Subito prima di ogni visita presso l'ambulatorio di pre-dialisi i pazienti saranno invitati a un colloquio con il farmacista della farmacia dell'ospedale, il quale somministrerà un

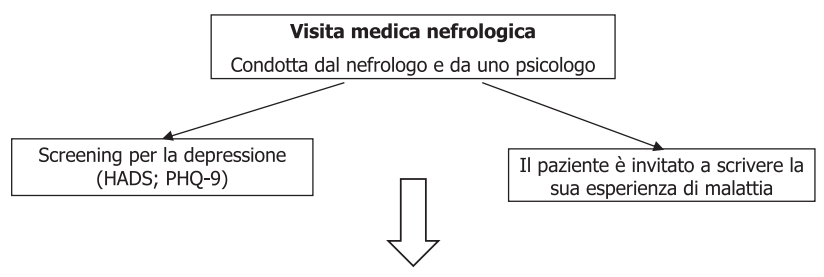

Intervento di counseling farmaceutico

- Screening aderenza alle terapie (Morinsky scale)

- Intervista semi-strutturata sull'aderenza alle terapie
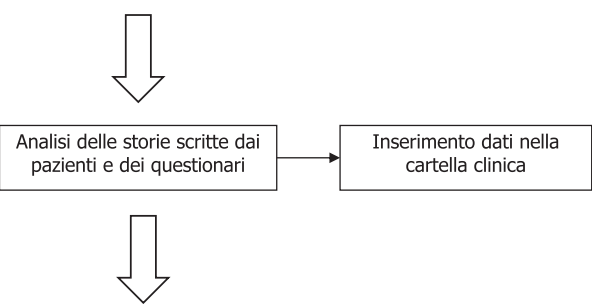

Visite mediche di follow-up

Discussione con il paziente dei risultati

Condivisione e decisione degli interventi da effettuare

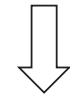

Incontri psico-educazionali di gruppo con pazienti e familiari (conduttori: nefrologo, psicologo, farmacista)

\begin{tabular}{|l|}
\multicolumn{1}{c|}{ Altri interventi: } \\
-Colloquio tra il paziente (ed eventualmente i suoi familiari), il \\
nefrologo e lo psicologo \\
- Psicoterapia: individuale, familiare, di gruppo \\
- Interventi di rete (es. MMG, assistente sociale)
\end{tabular}

Fig. 1 - Protocollo dell'intervento di Medicina Narrativa per pazienti con MRC.

questionario per l'aderenza alle terapie (Morisky Medication Adherence Scale) ed effettuerà un colloquio per esplorare atteggiamenti e comportamenti della persona verso le terapie;

4) restituzione al paziente e decision-making. Durante le successive visite di follow-up nefrologiche saranno discussi con i pazienti i risultati dell'analisi delle storie scritte e i risultati dei questionari. Nei casi di bassa aderenza alle terapie, saranno concordati possibili interventi per promuovere l'aderenza (per esempio, interventi psico-educazionali, psicoterapia, ecc.);

5) incontri psico-educazionali di gruppo. Ogni mese sarà svolto un incontro psico-educazionale di gruppo a partecipazione libera con i pazienti e i loro familiari, della durata di circa 1 ora e trenta/ 2 ore, condotto dal nefrologo, dallo psicologo e dal farmacista. Negli incontri saranno affrontate le principali tematiche legate alla MRC, alle terapie e ai comportamenti di salute (tra cui l'aderenza al percorso di cura). 
Durante i mesi del progetto, i pazienti saranno monitorati rispetto ai valori biochimici (controllo metabolico del calciofosforo, dell'anemia, della progressione dell'insufficienza renale, ecc.) e antropometrici (peso, pressione arteriosa). Saranno, inoltre, registrate le eventuali visite mediche o gli esami diagnostici saltati dai pazienti.

\section{Conclusioni}

In un suo famoso libro "Istruzioni per rendersi infelici" (21), lo psicologo austriaco Paul Watzlawick riporta questo aneddoto:

Sotto un lampione c'è un ubriaco che sta cercando qualcosa. Si avvicina un poliziotto e gli chiede che cosa abbia perduto. "Ho perso le chiavi di casa», risponde l'uomo, ed entrambi si mettono a cercarle.

Dopo aver guardato a lungo, il poliziotto chiede all'uomo ubriaco se è proprio sicuro di averle perse lì.

L'altro risponde:

"No, non le ho perse qui, ma là dietro», e indica un angolo buio in fondo alla strada.

"Ma allora perché diamine le sta cercando qui?»

«Perché qui c'è più luce!»

È difficile abbandonare i luoghi che ci hanno illuminato per anni. Luoghi a cui spesso ci rivolgiamo per cercare risposte a problemi nuovi, anche se questi necessitano di soluzioni metodologicamente diverse.

Seguendo questo ragionamento la medicina non può dare risposte alla complessità dei bisogni di pazienti cronici poliproblematici attingendo a un modus operandi del passato, benché questo possa aver avuto a suo tempo un razionale e prodotto indubbi risultati.

L'adesione del paziente con MRC ai trattamenti rientra a pieno titolo in questa sfera e possiamo dire che tale fenomeno costituisce una delle principali sfide nel campo della nefrologia clinica. Lasciando appunto al passato un modello medico-paternalistico in cui il soggetto malato aveva più una mera funzione passiva, le attuali tendenze, che la Medicina Narrativa sposa e arricchisce, devono prevedere il coinvolgimento attivo dei pazienti e dei loro familiari nel percorso di cura.

Possiamo parlare di una co-costruzione dinamica del percorso assistenziale tra paziente e medico in cui quest'ultimo risponde al duplice bisogno del malato di conoscere e capire "know and understand" e di essere compreso e conosciuto "feel known and understood" (22).

Il progetto pilota esposto in questo articolo vuole essere un tentativo di implementare, nella pratica clinica degli ambulatori di pre-dialisi, un protocollo basato su un approccio di Medicina Narrativa in cui i bisogni biologici, psicologici e sociali dei pazienti sono presi in considerazione da un team multi-professionale e discussi con i pazienti e i loro familiari.

\section{Disclosures}

Conflict of interest: The authors have no conflict of interest. Financial support: The authors declare no financial support.

\section{Bibliografia}

1. Hill NR, Fatoba ST, Oke JL,, et al. Global Prevalence of Chronic Kidney Disease - A Systematic Review and Meta-Analysis. PLoS One. 2016;11(7):e0158765.

2. De Nicola L, Donfrancesco C, Minutolo R, et al. Epidemiologia della MRC in Italia: stato dell'arte e contributo dello studio CHARES. G Ital Nefrol. 2011;28:(4):401-7.

3. Rosati A, Francesconi P, Profili F, Mennuti N. Prevalenza e rischi associati di mortalità ed eventi cerebro-cardio-vascolari in Toscana. Toscana Medica. Febbraio 2017.

4. Ruggenenti P, Perticucci E, Cravedi $P$, et al. Role of remission clinics in the longitudinal treatment of CKD. J Am Soc Nephrol. 2008;19(6)1213-24.

5. Turchetti G, Bellelli S, Amato M, et al. The social cost of chronic kidney disease in Italy. Eur J Health Econ. 2017;18(7):847-58.

6. Kerr M, Bray B, Medcalf J, O'Donoghue DJ, Matthews B. Estimating the financial cost of chronic kidney disease to the NHS in England. Nephrol Dial Transplant. 2012;27Suppl.3:iii73-80.

7. Durand-Zaleski I, Combe C, Lang P. International Study of Health Care Organization and Financing for end-stage renal disease in France. Int J Health Care Finance Econ. 2007;7(2-3):171-83.

8. Ministero della Salute. Documento di indirizzo per la malattia renale cronica 2014.

9. Mechta Nielsen T, Frøjk Juhl M, Feldt-Rasmussen B, Thomsen T. Adherence to medication in patients with chronic kidney disease: a systematic review of qualitative research. Clin Kidney J. 2018;11(4):513-27.

10. Burnier M, Pruijm M, Wuerzner G, Santschi V. Drug adherence in chronic kidney diseases and dialysis. Nephrol Dial Transplant. 2014;30(1):39-44.

11. Schmid H, Hartmann B, Schiffl $H$. Adherence to prescribed oral medication in adult patients undergoing chronic hemodialysis: a critical review of the literature. Eur J Med Res. 2009;14(5): 185-90.

12. Ellis R, Welch J. Medication-taking behaviours in chronic kidney disease with multiple chronic conditions: a meta-ethnographic synthesis of qualitative studies. J Clin Nurs. 2017;26(5-6): 586-98.

13. Mason NA. Polypharmacy and medication-related complications in the chronic kidney disease patient. Curr Opin Nephrol Hypertens. 2011;20:492-7.

14. Yu YJ, Wu IW, Huang CY, et al. Multidisciplinary predialysis education reduced the inpatient and total medical costs of the first 6 months of dialysis in incident hemodialysis patients. PLoS One. 2014;9(11):e112820.

15. Cukor D, Rosenthal DS, Jindal RM, Brown CD, Kimmel PL. Depression is an important contributor to low medication adherence in hemodialyzed patients and transplant recipients. Kidney Int. 2009;75(11):1223-9.

16. Cedillo-Couvert EA, Ricardo AC, Chen J, et al. Self-reported Medication Adherence and CKD Progression. Kidney Int Rep. 2018;3(3):645-51.

17. Cukor D, Peterson R, Cohen S, et al. Depression in end-stage renal disease hemodialysis patients. Nat Clin Prac Nephr. 2006;12:678-87. 
18. DiMatteo RM, Lepper HL, Croghan TW. Depression is a risk factor for noncompliance with medical treatment. Arch Intern Med. 2000;160:2101-7.

19. Cukor D, Ver Halen N, Asher DR. Psychosocial intervention improves depression, quality of life, and fluid adherence in hemodialysis. J Am Soc Nephrol. 2014;25:196-206.

20. De Geest S, Sabaté E. Adherence to long-term therapies: evidence for action. Eur J Cardiovasc Nurs. 2003;2(4):323.
21. Watzlawick P. Istruzioni per rendersi infelici. Feltrinelli Editore. 1997.

22. van Vliet LM, Epstein AS. Current state of the art and science of patient-clinician communication in progressive disease: patients' need to know and need to feel known. J Clin Oncol. 2014;32(31):3474-8. 